\title{
Kinetics of in vitro bronchoconstriction in an elastolytic mouse model of emphysema
}

\author{
M.A. Khan, S. Kianpour, M.R. Stämpfli and L.J. Janssen
}

\section{ABSTRACT: Thin-slice videomicroscopy was used to examine the kinetics of constriction in small airways in situ.}

Balb/C mice inhaled elastase (0-20 IU), and were then left to recover for 14 days before euthanisation and lung removal. Cholinergic responsiveness was assessed in thin lung slices. Magnitude and velocity of narrowing in response to $10^{-5} \mathrm{M}$ acetylcholine (ACh), as well as the full concentration-response relationship for $\mathrm{ACh}\left(10^{-8}-10^{-5} \mathrm{M}\right)$ were assessed.

In vivo exposure to elastase was accompanied by statistically significantly decreased magnitudes and velocities of contraction, but no change in the $\mathrm{ACh}$ concentration-response relationship. Conversely, overnight, in vitro exposure of slices from control animals to elastase $\left(2.5 \mu \mathrm{g} \cdot \mathrm{mL}^{-1}\right)$ resulted in increased magnitudes and velocities of airway narrowing, with impaired relaxation, as well as marked tearing of the airways from the surrounding parenchyma. These changes are characteristic of decreased tethering forces on the airway wall.

Thus, the lung slice technique coupled with videomicroscopic analysis of airway contraction velocities provides a powerful tool to study airway-parenchymal interactions. The elastolytic model of emphysema, which manifests with airspace enlargement and loss of parenchymal attachments, is accompanied by decreased airway contraction kinetics. The mechanism(s) underlying this loss of function remain to be elucidated.

KEYWORDS: Airway smooth muscle, cholinergic, collagen, connective tissue, contraction, elastin

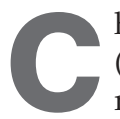
hronic obstructive pulmonary disease (COPD), characterised by airways inflammation, bronchitis and lung remodelling (both emphysematous and fibrotic changes) [1$3]$, is already one of the major causes of mortality and morbidity across the world, and is increasing in prevalence $[4,5]$. The major triggering factor is cigarette smoke, which accounts for $80-90 \%$ of COPD cases. However, in the population of smokers, only $15 \%$ of the subjects develop chronic airflow limitations [6]. Several serine proteases and matrix metalloproteases are expressed in association with COPD in humans [7]. Also, many different experimental approaches (intranasal instillation of elastase, gene-targeted upregulation of elastase expression or downregulation of $\alpha$ antitrypsin, use of various protease inhibitors) have highlighted important roles for endogenous proteases in airspace enlargement, and have identified many interactions between these proteolytic systems [7]. In addition to playing a key role in destruction of the extracellular matrix, particularly elastin, proteases may also be important in regulating inflammation through the generation

For editorial comments see page 611. of chemokines and cytokines, and by blazing trails for cells through tissue barriers [7].

Awareness of this central or even causal role of proteases has led to the development of several rodent models of COPD [8], including one in which the animals are made to inhale porcine pancreatic elastase intranasally [9, 10]. This challenge results in an inflammatory response which resolves within days, as well as structural changes which persist for at least 100 days. STONE et al. [11] have previously reported the dosedependent effects of elastase in hamsters; even doses as low as 6 units of elastase produced mild emphysema, as detected by pulmonary function testing. Likewise, Gray and Mitchell [12] described the effects of intramural injection of elastase in isolated bronchial segments of pigs; this led to uncoupling of the airway wall from the parenchyma, resulting in increasing responsiveness in vitro. QIAN and MITZNER [13] reported that emphysematous parenchyma showed greater sensitivity to acetylcholine (ACh) compared with control parenchyma and significantly greater maximal contractility to $\mathrm{ACh}$ in the elastaseinduced hamster model of emphysema.
AFFILIATION

Asthma Research Group, Firestone Institute for Respiratory Health, St. Joseph's Hospital, and Depts of Medicine and Pathology, McMaster University, Hamilton, ON, Canada.

CORRESPONDENCE

L.J. Janssen

L-314

St. Joseph's Hospital

40 Charlton Avenue East Hamilton

ON

L8N 4A6 Canada

Fax: 19055406510

E-mail: janssenl@mcmaster.ca

Received:

March 052007

Accepted after revision:

May 202007

SUPPORT STATEMENT

The present study were supported by a grant-in-aid provided by the Canadian Institutes of Health Research.

STATEMENT OF INTEREST A statement of interest for this study can be found at www.erj.ersjournals.com/misc/ statements.shtml 
Using this approach, it has been possible to examine and quantify various histological and immune/inflammatory indices associated with experimentally induced emphysema. However, COPD is also characterised by partially reversible airflow obstruction, atelectasis and gas trapping, and airway hyperresponsiveness, all contributing to compromised gas exchange in the lung [14,15]. These aspects of COPD and emphysema have proven harder to examine; changes in airway function per se are particularly difficult to examine. Although overall airflow and resistance can be measured either in vivo or in excised lungs, it is not easy to attribute changes to specific segments of the airways, i.e. the larger airways versus the various bronchiolar vessels (conducting, terminal or respiratory). Imaging techniques, such as positron emission tomography or computer tomography, have allowed for discrimination of some of the larger of these airways in living animals, but these approaches are limited by temporal and spatial resolution, and require costly and sophisticated equipment. The recent introduction of the thin lung slice technique may overcome some of these barriers. Using this latter approach, the lungs are inflated with a gel-like substance, then cut into slices as thin as $50 \mu \mathrm{m}$, which can then be studied using standard videomicrometric techniques and the responsiveness of even terminal bronchioles monitored at video rates while still situated in their native environment (i.e. with parenchymal attachments largely intact). This approach has been used to examine airway wallparenchyma interactions in a rat model of allergen-induced airway hyperresponsiveness [16, 17], but not the elastolytic model of COPD described above.

The present authors combined this latter approach with a murine model of emphysema in order to explore the effects of parenchymal destruction on airway smooth muscle contraction kinetics. Balb/C mice were used, since these show a robust reproducible response to cholinergic stimulation when studied using the thin lung slice technique [18]. The current authors have added velocity measurements in ACh-induced contraction to better understand the effect of elastase treatment on the interactions of the airway with the parenchyma and the effects of changes in tethering imposed by surrounding parenchyma. The present authors hypothesised that decreased tethering might manifest as greater magnitude of narrowing, faster velocity of contraction and slower velocity of relaxation (the latter being particularly evident in taking the ratio of the two velocities).

\section{MATERIALS AND METHODS}

\section{Animal handling and in vivo delivery of elastase}

All experiments were approved by the Animal Research Ethics Board of McMaster University (Hamilton, ON, Canada) and carried out according to the guidelines of the Canadian Council on Animal Care.

Female Balb/C mice (6-8 weeks old) were purchased from Charles River Laboratories (Montreal, PQ, Canada) and maintained under specific pathogen-free conditions in an access-restricted area, on a 12-h light-dark cycle, with food and water provided ad libitum. One group of animals was anaesthetised with isofluorane according to institutional guidelines, and 0-20 IU of elastase/100 g body weight administered intranasally ( $15 \mu \mathrm{L}$ to each nostril) before returning them to the facility for recovery for 14 days. Otherwise, elastase-exposed and naive animals were sacrificed by carbon dioxide inhalation in a closed chamber for $1 \mathrm{~min}$ and lungs removed for use in histological or physiological studies as described below.

\section{Lung slicing}

Lungs were sliced using the protocol first described by BERGNER and SANDERSON [19] and adapted within the laboratory. Briefly, following euthanisation, the chest wall was removed, the trachea cannulated using an intravenous catheter (20G Intima, Becton Dickinson, Sandy, UT, USA), and the lungs inflated with $\sim 1.2 \mathrm{~mL}$ of agarose (type VII-A low gelling temperature; Sigma Aldrich, St. Louis, MO, USA) warmed to $37^{\circ} \mathrm{C}$; unless indicated otherwise, the latter was made up to a concentration of $2 \%$ in Hanks' buffered saline solution (HBSS). Air $(\sim 0.1-0.2 \mathrm{~mL})$ was subsequently injected in order to flush the agarose-HBSS out of the airways of interest (those which would be visualised) so that they would be free to contract and not subject to intraluminal resistance. The agarose was gelled by cooling the lung preparation to $4^{\circ} \mathrm{C}$ for $5-10 \mathrm{~min}$, after which the left lobe was removed and sliced (120- $\mu \mathrm{m}$ thickness) using an EMS-4000 tissue slicer (Electron Microscope Sciences, Fort Washington, PA, USA) at $4^{\circ} \mathrm{C}$. The slices were washed in HBSS and inspected under a phase-contrast microscope; those slices which were not torn or otherwise damaged were then incubated overnight in Dulbecco's modified Eagle's medium (DMEM) supplemented with antibiotics and antimycotics at $37^{\circ} \mathrm{C}$ and $5 \%$ carbon dioxide.

\section{In vitro measurement of airway contraction}

Lung slices were taken out after overnight incubation and transferred to fresh HBSS for $30 \mathrm{~min}$. Those which possessed airways that nearly filled the charge-coupled device (CCD) camera imaging area $(\sim 125 \mu \mathrm{m}$ outer diameter $)$ and which displayed an intact epithelium and parenchyma, as well as active epithelial ciliary beating, and which were not plugged with agarose were selected. Slices which met these criteria were mounted on a glass cover slip $(45 \times 50 \mathrm{~mm}$; Fisher Scientific, Suwanee, GA, USA), and held in position by a piece of nylon mesh (250 $\mu \mathrm{m}$ mesh; CMN-0250D, Small Parts, Miami Lakes, FL, USA), as well as a second glass cover slip $(22 \times 40$ $\mathrm{mm}$ ); this did not appear to offer any frictional opposition to movement of the airway (it appeared unconstrained to move over the glass surface, although this was not specifically measured). DMEM was superfused between the two cover slips and over the tissue slice at a rate of $\sim 4.5 \mathrm{~mL} \cdot \mathrm{min}^{-1}$. ACh was added/removed via this perfusate and had excellent access to the airway of interest, as indicated by a latency for onset of the cholinergic response of $<5 \mathrm{~s}$. Phase-contrast images were acquired with a high-resolution CCD solid state video camera (CV-252; Nikon, Tokyo, Japan), and recorded in time lapse (3-ms exposure, 0.2 frames $\cdot \mathrm{s}^{-1}$ ) using image acquisition software (Video Savant; IO Industries, London, ON, Canada). Video images were transformed to recordings of airway area versus time using Scion image analysis software (Scion, Frederick, MD, USA), which converted the 10-bit video images to binary and then measured cross-sectional area of the airway in each frame by pixel summing. Finally, the recordings were analysed and statistical comparisons made.

\section{Histology}

The trachea of each excised lung was cannulated with a polyethylene tube (Becton Dickinson, Sparks, MD, USA) and 
inflated with $10 \%$ formalin at a constant pressure of $20 \mathrm{cmH}_{2} \mathrm{O}$. Lungs were fixed for $24 \mathrm{~h}$ and embedded in paraffin. Threemicrometre-thick sections were stained with haematoxylin and eosin. Mean chord length was assessed as described previously [20]. Five random fields per lung were digitised using a Leica DMR microscope (Leica Microsystems, Wetzlar, Germany). Sections were analysed using Leica Qwin Image Processing Software (Leica Imaging Systems, Cambridge, UK). Large airways and bronchi were excluded from the analysis. Data are shown as mean chord length of the airspace, a measure independent of the thickness of alveolar septa.

\section{In vitro exposure to elastase}

Where specifically indicated, slices from naive animals (those which did not inhale elastase in vivo) were incubated overnight with porcine pancreatic elastase (Sigma Aldrich) at a final concentration of $2.5 \mu \mathrm{g} \cdot \mathrm{mL}^{-1}$ in DMEM supplemented with antibiotics and antimycotics at $37^{\circ} \mathrm{C}$ and $5 \%$ carbon dioxide. After overnight incubation, slices were analysed under a phasecontrast microscope and airways with minimal visible damage were selected for assessment of cholinergic responsiveness.

\section{Materials}

Cell culture reagents were obtained from Invitrogen Life Technologies (GIBCO, Carlsbad, CA, USA). DMEM for slice incubation was supplemented with PennStrep (penicillin $10,000 \mathrm{U} \cdot \mathrm{mL}^{-1}$, streptomycin $10,000 \mathrm{U} \cdot \mathrm{mL}^{-1}$ ), amphotericin $\mathrm{B}$ $(125 \mu \mathrm{g}), \mathrm{L}-$ ascorbic acid $\left(35 \mu \mathrm{g} \cdot \mathrm{mL}^{-1}\right)$, transferrin $\left(5 \mu \mathrm{g} \cdot \mathrm{mL}^{-1}\right)$, selenium $\left(3.25 \mathrm{ng} \cdot \mathrm{mL}^{-1}\right)$ and insulin $\left(2.85 \mu \mathrm{g} \cdot \mathrm{mL}^{-1}\right)$. HBSS in most cases was supplemented with hydroxyethyl piperazine ethane sulphonic acid buffer (0.2 M pH 7.4). All other reagents
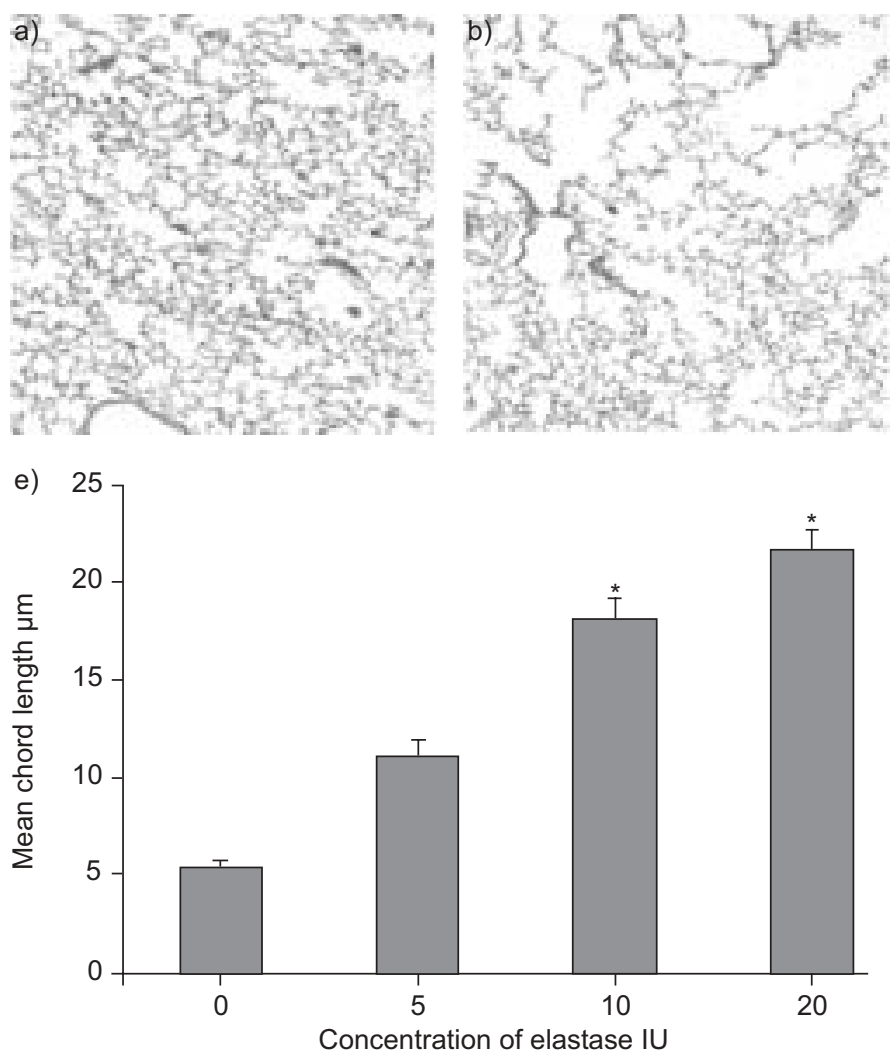

were obtained from Sigma unless otherwise specified. Porcine pancreatic elastase (Sigma Aldrich) was dissolved in $0.9 \%$ sodium chloride at a concentration of $1 \mathrm{mg} \cdot \mathrm{mL}^{-1}$, divided into $250-\mu \mathrm{L}$ aliquots and stored at $-20^{\circ} \mathrm{C}$.

\section{Statistics}

Airway areas were standardised as a percentage of lumen area measured at the beginning of the experiment (immediately prior to challenge with the first bolus dose of $\mathrm{ACh}$ ). The first derivative of such recordings of airway area was taken to obtain the velocities of narrowing or dilation. The velocities of smooth muscle shortening were also obtained by using area measurements to estimate the radius of the airway (assuming the latter to be circular; area $=\pi \times r^{2}$, where $r$ is the radius), followed by use of the radius to calculate circumference as the product of $\pi$ and diameter. In this way, it was possible to estimate the changes in length on a frame-by-frame basis, and the first derivative of this as the velocity of shortening. All data are expressed as mean \pm SEM. Statistical comparisons between different groups were carried out using unpaired ANOVA; an unpaired t-test was used for comparing velocities of lumen narrowing in the same tissue. Correlation analysis was carried out using Pearson's test. A p-value $<0.05$ was considered statistically significant.

\section{RESULTS}

\section{In vivo elastase treatment causes airspace enlargement}

In an initial set of experiments, the present authors quantified the degree of airspace enlargement induced by various concentrations of elastase given intranasally (from 0.02-20 IU elastase per $100 \mathrm{~g}$ body weight) 2 weeks prior to removal of
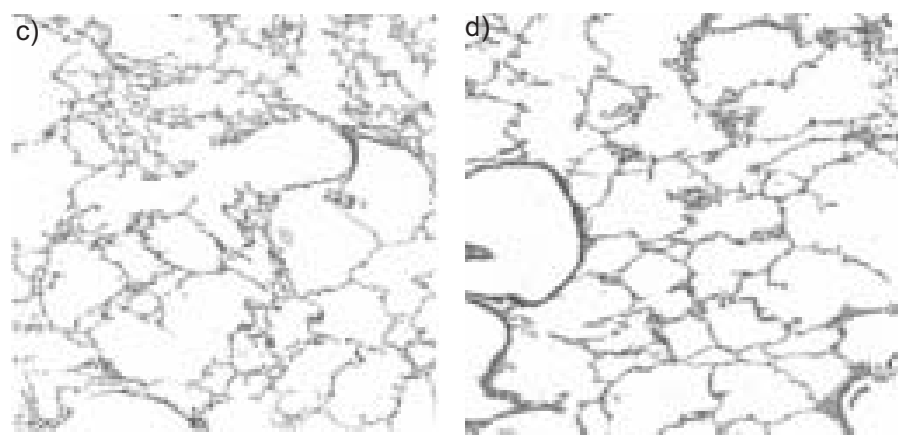

FIGURE 1. Lung slices from a) a naïve lung for comparison and mice inoculated intranasally with b) 5, c) 10 or d) $20 \mathrm{IU}$ porcine pancreatic elastase per $100 \mathrm{~g}$ body weight. e) Airspace enlargement was quantified and expressed as mean chord length. Data are shown as mean \pm SE. $n=3$. *: $p<0.05$, ANOVA. 
the lungs. Figure 1 shows that 20 and $10 \mathrm{IU}$ per $100 \mathrm{~g}$ body weight induced widespread emphysematous lesions. Only minimal and focal airspace enlargement was observed at 5 IU per $100 \mathrm{~g}$ body weight. No tissue damage was observed at concentrations $<2$ IU per $100 \mathrm{~g}$ body weight (data not shown). Quantification of airspace enlargement (fig. 1e) showed statistically significantly increased mean chord length at 20 and $10 \mathrm{IU}$ per $100 \mathrm{~g}$ body weight $(\mathrm{p}<0.05)$.

\section{Cholinergic responses in control tissues}

The experimental protocol that was used to assess airway responsiveness to cholinergic stimulation is shown in figure 2. Tissues were first challenged with $10^{-5} \mathrm{M}$ ACh to evoke a maximal constrictor response; in naive tissues, this elicited a rapid and dramatic decrease in airway diameter which reached a peak within $5 \mathrm{~min}$. ACh was then washed from the tissues by superfusing with regular HBSS for 5-10 min, during which time the airway relaxed back toward its baseline diameter. Finally, the full cholinergic concentration-response relationship was explored by challenging the tissues with $\mathrm{ACh}$ at concentrations ranging from $10^{-8}$ to $10^{-5} \mathrm{M}$.

Figure $2 \mathrm{~d}$ summarises the velocities of narrowing/dilation, derived on a frame-by-frame basis from the first derivative of the lumen area recording, in the same representative lung slice. The velocity of narrowing (an active process) in this trace was more than twice as fast as that for relaxation (a passive process). The ability of this approach to discriminate different contraction velocities is also highlighted in the second portion of the trace, in which the responses to increasing concentrations of ACh are shown. In particular, both $10^{-7} \mathrm{M}$ ACh and $10^{-6} \mathrm{M}$ ACh evoked an $\sim 20 \%$ decrease in airway area, but the latter did so with a velocity almost seven times greater than that of the former. Alternatively, it is possible to estimate velocities of smooth muscle shortening per se, by converting changes in lumen area to changes in lumen circumference (see the Methods section). The velocities of shortening were found to be nearly identical to those of lumen narrowing, differing only slightly at extremely small airway diameters (see figs $2 \mathrm{~d}$ and $6 \mathrm{~d}$ ), indicating that monitoring changes in airway area is a useful surrogate for following changes in smooth muscle length. That notwithstanding, given that the airways were not perfectly circular, velocities of lumen narrowing were used in statistical comparisons.

On average, $10^{-5} \mathrm{M}$ ACh evoked a mean decrease in initial airway area of $53.6 \pm 3.12 \%(n=5$; fig. $3 \mathrm{~g})$, and the velocity of contraction (fig. $3 \mathrm{~h}$ ) was statistically significantly greater than that of relaxation (fig. $3 \mathrm{i} ; \mathrm{p}<0.05$ ); the mean ratio of these two velocities was $4.14 \pm 0.69$ (fig. 3 j). The mean cumulative ACh concentration-response relationship in the control tissues shown in figure 3 is given in figure 4 .

\section{Validation of the experimental approach}

The impact of several different experimental parameters on the measurements made was assessed before employing this novel approach to examine the effects of altered tethering forces on airway function.

BAI et al. [21] have shown that the magnitudes of constrictor responses in lung slices vary over a very wide range of airway sizes; however, they did not include contraction/relaxation
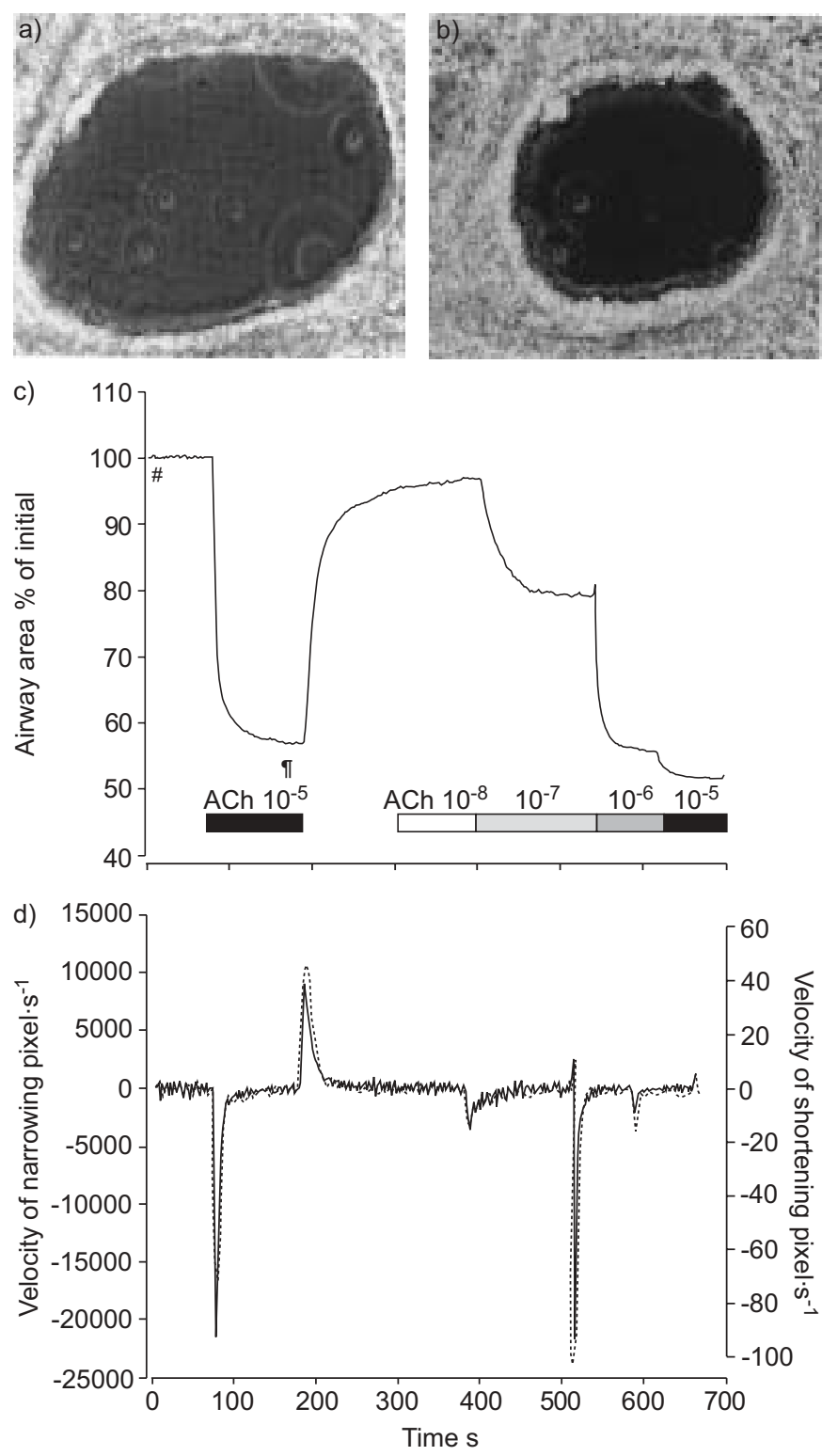

FIGURE 2. Single-frame images of the airway a) before and b) during stimulation with acetylcholine (ACh; $\left.10^{-5} \mathrm{M}\right)$. c) Measurements of airway diameter during bolus addition of a supramaximally effective concentration of $A C h\left(10^{-5} \mathrm{M}\right)$, followed by washout of $\mathrm{ACh}$, and then re-introduction in log unit increments beginning at $10^{-8} \mathrm{M}$. ${ }^{\#}$ and " correspond to images in a) and b), respectively. d) First-order derivatives of data in c), giving velocities of lumen narrowing ( - ) or of airway smooth muscle shortening (-------); these were obtained by estimating lumen radius on a frame-by-frame basis (see the Methods section). Tissue was obtained from a naive mouse, and was incubated overnight in Dulbecco's modified Eagle's medium.

velocities in those comparisons. In the present study, in which a much smaller range of airway sizes was examined, neither the magnitudes nor the velocities of contraction were found to correlate in a statistically significant manner with airway size (fig. 5; $\mathrm{p}>0.05$, Pearson's correlation test).

To address whether the agarose used to inflate the lungs might affect the mechanical responses being measured [16], the 

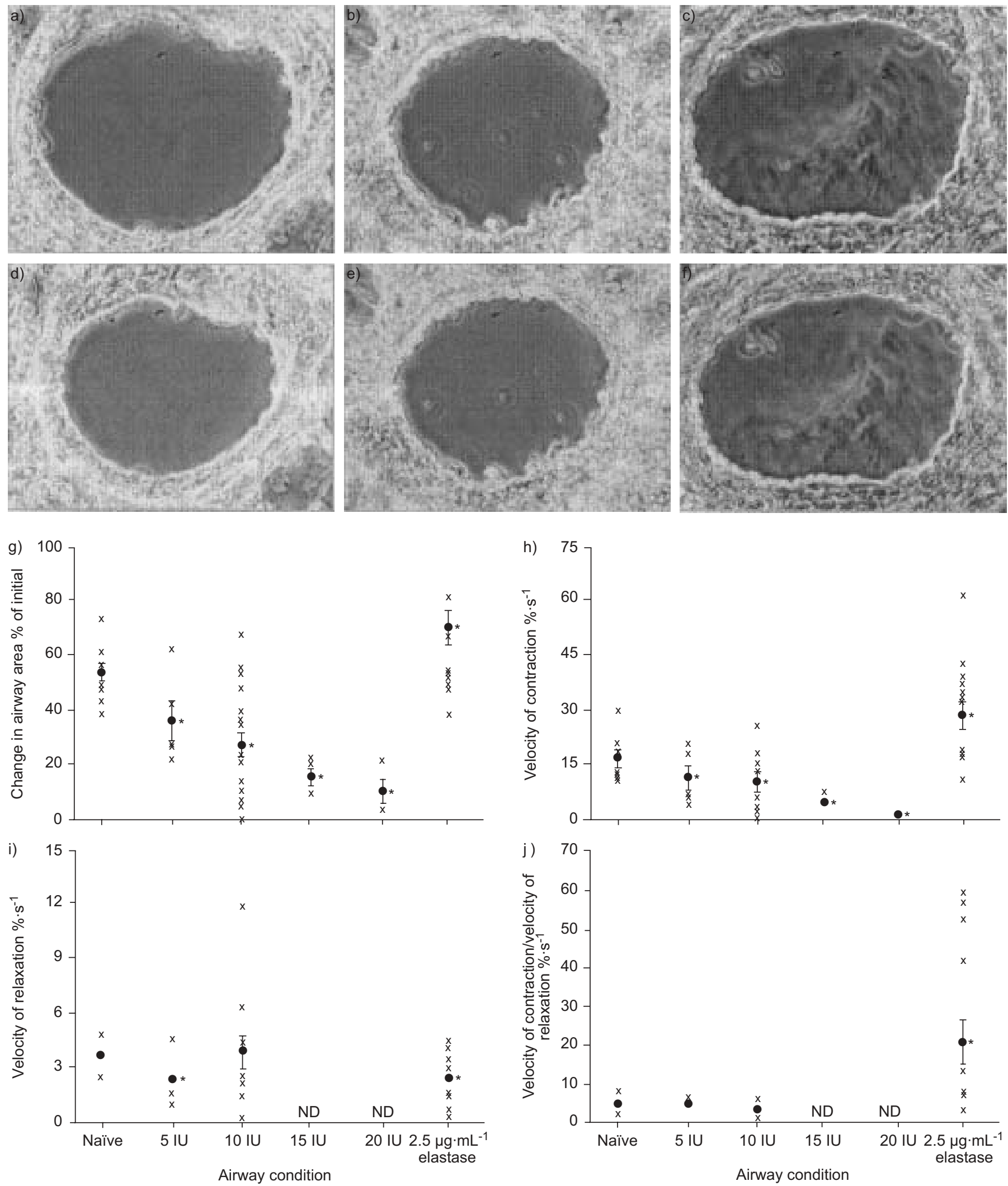

FIGURE 3. Effect of elastase on cholinergic mechanical responses. a-f) Representative images of airways treated with a, b, d, e) 10 IU or c, f) 20 IU elastase in vivo, before (at rest; $\mathrm{a}-\mathrm{c}$ ) and during challenge with acetylcholine $\left(10^{-5} \mathrm{M} ; \mathrm{d}-\mathrm{f}\right)$. g) Summary of the magnitudes of contraction, h) the velocities of contraction, i) the velocities of relaxation, and j) the ratios of the two velocities in airway slices from naive mice and those treated with elastase either in vivo $(5,10,15$ or $20 \mathrm{IU})$ or in vitro $\left(2.5 \mu \mathrm{g} \cdot \mathrm{mL}^{-1}\right)$, as indicated. ND: not determined. $\bullet$ : mean \pm SE $(n>5$ for all slices). $x$ : data from individual slices. *: $p<0.05$, ANOVA. 


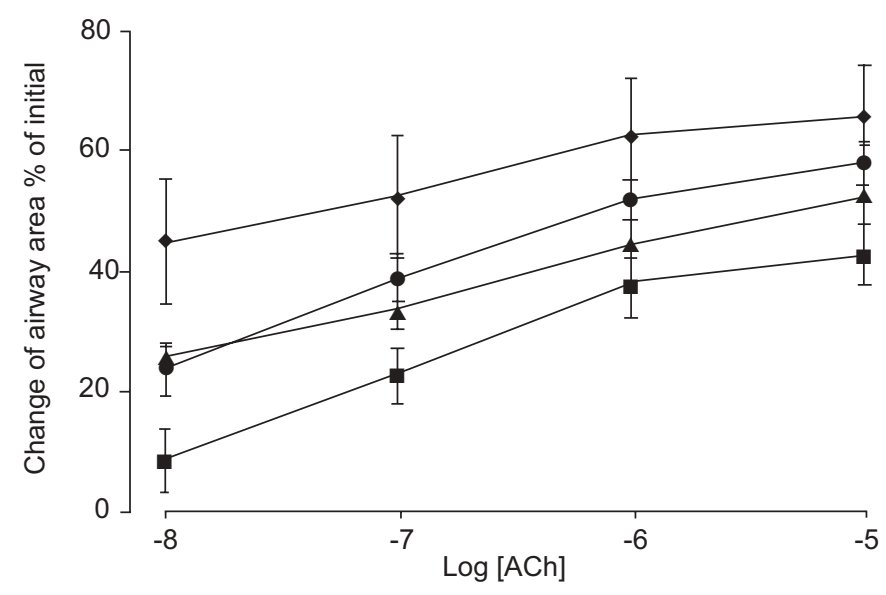

FIGURE 4. Mean concentration-response relationship for acetylcholine (ACh) obtained in lung slices from naive mice $(\bullet)$ and those treated in vivo with elastase (5 (匹) or $10 \mathrm{IU}(\mathbf{\Lambda})$ ), as well as naive slices incubated overnight with $2.5 \mu \mathrm{g} \cdot \mathrm{mL}^{-1}$ elastase ( ), as indicated. $\mathrm{n}>5$ in each case.

concentration of agarose was halved: this was found to have no statistically significant effect on either the magnitudes or velocities of contraction or relaxation (table 1).

Lung compliance measured in whole animal studies varies with extent of lung inflation. Using the thin lung slice technique, the lungs are inflated to full physiological lung capacity with $\sim 1.2 \mathrm{~mL}$ of agarose, depending on the relative size of the animal. The dependence of the present study's measurements was evaluated on the degree of lung inflation. Even when the volume of agarose injected was decreased by one-third, no statistically significant effect was observed on either the magnitude or velocities of the measured responses (table $1 ; \mathrm{p}>0.05$, unpaired $\mathrm{t}$-test).

\section{Effect of in vivo elastase treatment on cholinergic responsiveness}

When tissues from animals that had inhaled elastase in vivo were examined using the experimental protocol described above, it was found that elastase treatment had a consistent, profound and statistically significant effect on the responses to cholinergic stimulation, to a degree that was dependent upon the concentration of elastase used. In particular, the mean magnitude of the response to the first bolus challenge with ACh was decreased, even in those tissues that had received 5 IU elastase in vivo, and were nearly abolished in those treated in vivo with 15 or 20 IU elastase (fig. 3g; $<<0.05$, ANOVA). Not surprisingly, the mean cholinergic response in tissues pretreated in vivo with $10 \mathrm{IU}$ elastase was intermediate between these two extremes; however, it was noted with interest that the responses in individual tissues ranged from being apparently normal (similar to those in naive tissues) to being as severely affected as the 15 and 20 IU elastase-treated tissues.

The velocities of contraction and relaxation were also found to be significantly decreased ( $p<0.05$, ANOVA) compared with naive animals in tissues from animals that had inhaled elastase in vivo, and that the degree of inhibition was dependent on the concentration of elastase (fig. $3 g-3 j$ ).
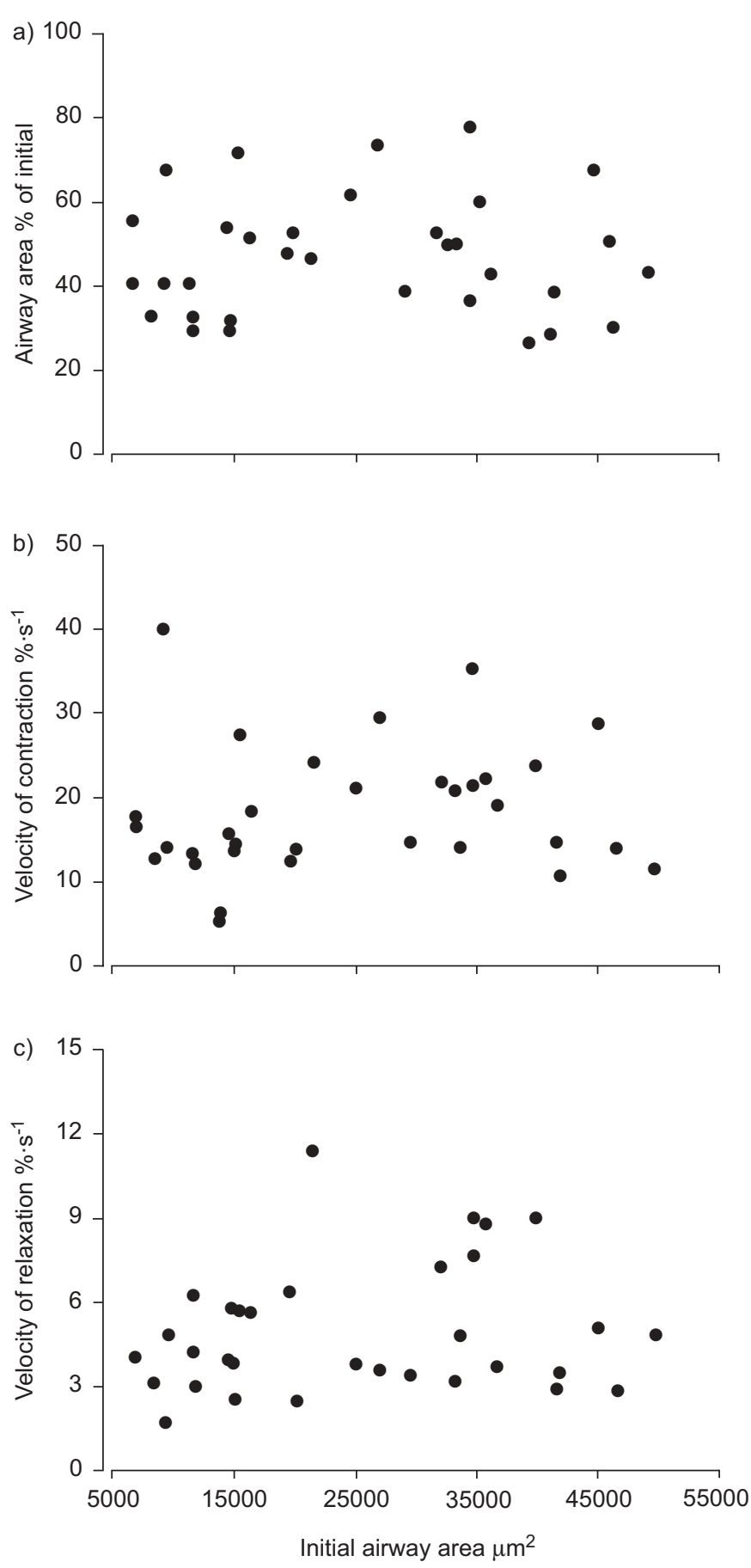

FIGURE 5. a) Magnitudes of contraction, and velocities of b) contraction and c) relaxation. These did not correlate with initial airway size.

Finally, the ACh concentration-response relationship was examined in tissues pre-exposed in vivo to 5 or $10 \mathrm{IU}$ elastase (the marked inhibitory effect of 15 and 20 IU elastase on the responses to maximal cholinergic stimulation precluded investigations of submaximal cholinergic responses). The mean magnitudes of the responses to $10^{-8}$ to $10^{-5} \mathrm{M}$ ACh are given in figure 4. No statistically significant effect of elastase in vivo (5 or $10 \mathrm{IU})$ was observed on the cholinergic concentrationresponse relationship (ANOVA). 


\begin{tabular}{|c|c|c|c|}
\hline 1 & $45 \pm 4.3$ & $16.5 \pm 3.0$ & $3.3 \pm 0.4$ \\
\hline 2 & $53.6 \pm 3.12$ & $18.3 \pm 2.6$ & $3.5 \pm 0.5$ \\
\hline \multicolumn{4}{|c|}{ Volume of agarose $\mathrm{mL}$} \\
\hline 1.0 & $43.6 \pm 4.5$ & $12.8 \pm 1.9$ & $4.7 \pm 0.8$ \\
\hline 1.1 & $46.2 \pm 3.1$ & $14.4 \pm 1.2$ & $5.7 \pm 1.2$ \\
\hline 1.2 & $45 \pm 3.6$ & $17.5 \pm 2.3$ & $6.5 \pm 1.1$ \\
\hline
\end{tabular}

\section{Effect of in vitro elastase treatment on cholinergic responses}

In order to address the effects of simple destruction of elastic tissues without the confounding influences of inflammation, fibrosis and repair, lung slices from another group of naive animals were incubated overnight with varying concentrations of elastase added to the culturing medium, then examined the next day using the experimental protocol described above. Slices pre-treated overnight in vitro with $100 \mu \mathrm{g} \cdot \mathrm{mL}^{-1}$ were very soft and fragile, with patchy parenchyma, epithelial degeneration and noticeably less epithelial ciliary beating activity. Those treated with $50 \mu \mathrm{g} \cdot \mathrm{mL}^{-1}$ elastase had active cilia, but the parenchyma was also patchy and fragile. Slices pre-treated overnight with 5 and $2.5 \mu \mathrm{g} \cdot \mathrm{mL}^{-1}$ elastase had epithelium that was normal in appearance and less parenchymal damage (fig. 6c). The present authors elected to use $2.5 \mu \mathrm{g} \cdot \mathrm{mL}^{-1}$ elastase when studying the effects of overnight in vitro elastase treatment on cholinergic responsiveness.

Slices treated in this way exhibited a larger and much more brisk contractile response to $\mathrm{ACh}$ and a considerably more sluggish relaxation upon washout of $\mathrm{ACh}$ (see representative tracings in figs $6 a$ and b). In fact, the airway often did not return to its baseline diameter, perhaps because the airways had torn away from their parenchymal attachments during the constrictor response. The mean magnitudes and velocities of this response are given in figure 3; one-way ANOVA revealed these to be statistically increased following in vitro elastase treatment, relative to control tissues. In contrast, the velocity of relaxation was significantly suppressed $(p<0.05)$.

The ACh concentration-response relationship was also altered by this direct overnight elastase treatment. Although $10^{-8} \mathrm{M}$ ACh was sub-threshold in the tissues described above, it now elicited a profound, albeit transient, contractile response (fig. 6). Likewise, responses to $10^{-7} \mathrm{M}$ ACh were significantly augmented. The mean concentration-response relationship is given in figure 4 .

\section{DISCUSSION}

Videomicrometry of lung slices has been used extensively to study a wide variety of mechanical responses [16, 17, 22, 23]. Others studies have used $0.75-1 \%$ agarose to inflate rodent lungs prior to cutting into slices $500-1000-\mu \mathrm{m}$ thick [16], or 250$\mu \mathrm{m}$ thick [22]. This approach was then modified using $2 \%$ agarose to inflate and stiffen the lungs prior to cutting into slices $\sim 100-\mu \mathrm{m}$ thick [19]. ADLER et al. [24] have previously demonstrated that $2 \%$ agarose-filled lungs have a shear modulus similar to that of air-filled lungs.

However, these earlier studies have, by and large, examined only the absolute magnitudes of airway narrowing, paying little or no attention to the dynamics of those changes. Important information pertaining to the connective tissue matrix is likely to be present in the time-course of airway narrowing/opening. In the present study, the technique has been extended to include estimations of instantaneous velocities of narrowing, and showed that the latter are a facile and close estimate of the velocities of smooth muscle shortening. This technique has another advantage over other in vitro techniques for measuring airway function; the latter are carried out under either isotonic or isometric conditions, whereas the airways in the lung slices and in the lungs in vivo contract auxotonically (neither isometrically nor isotonically).

For all these reasons, the present approach could prove to be invaluable in studying the interactions between the airway smooth muscle (ASM) and its surrounding parenchymal matrix. DUGUET et al. [25] have reported that airway constriction and velocity of narrowing is governed by the elastic load on the airway wall. The primary elastic loads that limit ASM shortening are the lung parenchyma (via the alveolar attachments to the airway wall), the airway wall itself and the internal resistance to shortening of the smooth muscle cell [26-28].

COPD is characterised in part by lung parenchymal destruction and enlargement of the air spaces with loss of functioning alveoli. There is considerable evidence that this parenchymal destruction is secondary to an excess of proteolytic activity, which in turn is related to airway inflammation [29, 30]. Many of these changes are reproduced in murine models of elastaseinduced parenchymal destruction. However, while the histological and inflammatory indices referred to above are easily examined in rodent models, the changes in airway function brought on by altered tethering forces, which are also highly clinically relevant, are not so easily examined. The thin lung slice technique offers the opportunity to easily monitor mechanical responses of even very small airways with high temporal and spatial resolution while they are still in their native environment.

The present authors hypothesised that parenchymal destruction in the present rodent model of emphysema would manifest as larger and faster contractions, with slowed airway 

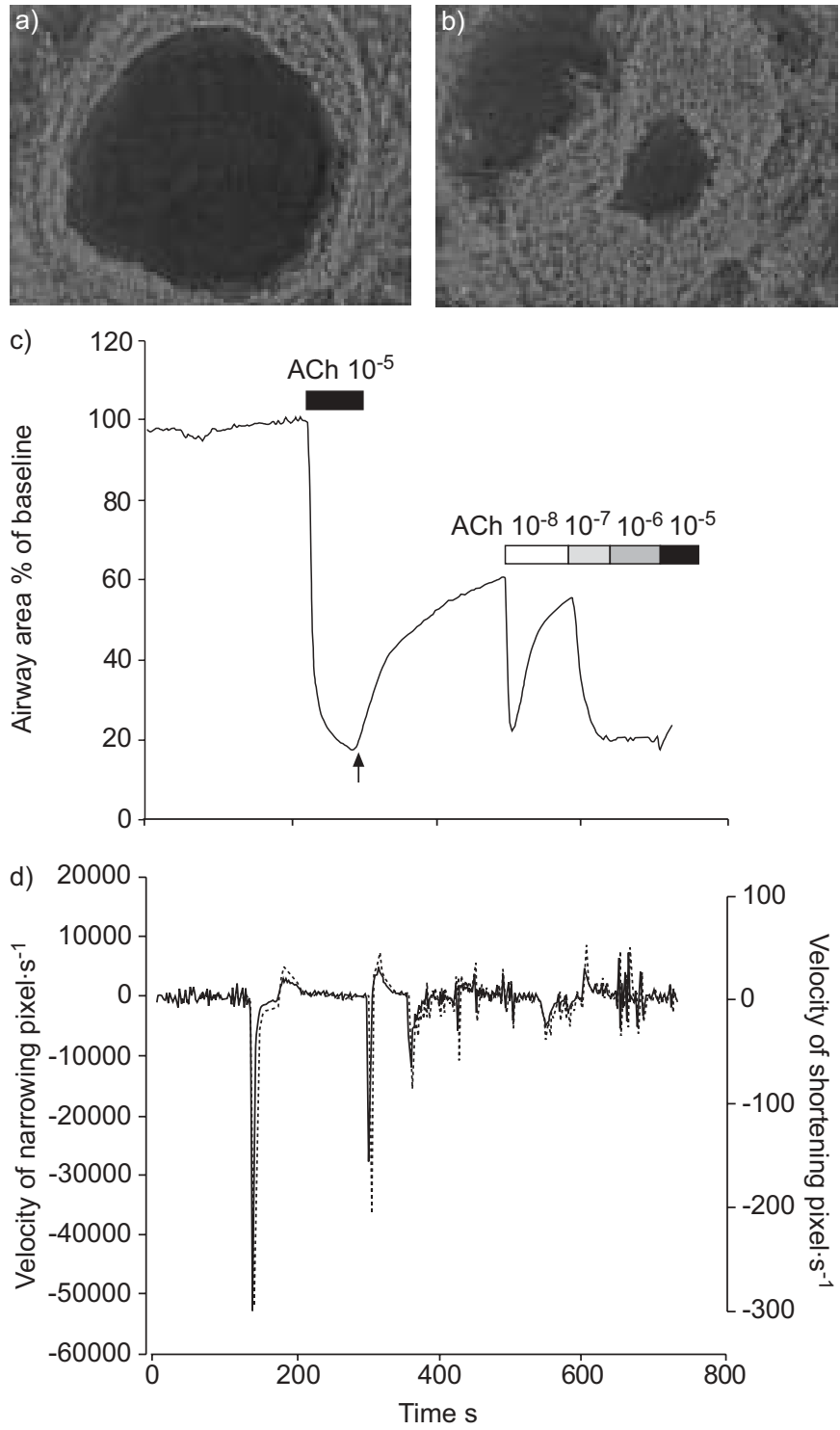

FIGURE 6. Individual frames showing a naïve lung slice incubated overnight with Dulbecco's modified Eagle's medium containing $2.5 \mu \mathrm{g} \cdot \mathrm{mL}^{-1}$ elastase a) immediately before application of bolus concentration of acetylcholine ( $\mathrm{ACh}$; $\left.10^{-5} \mathrm{M}\right)$ and $\mathrm{b}$ ) at the peak of that contraction. Note the tearing of the airway away from the parenchyma. c) and d) Raw tracings of airway diameters and velocity of contraction, respectively. —_ Velocity of narrowing; - - - -: velocity of shortening. The arrow indicates when the Hanks buffered saline solution washing occurred.

re-opening upon induction of relaxation. Contrary to expectations, the present authors found the cholinergic responses in the animals treated in vivo with elastase to be consistently and markedly decreased compared with matched naive animals. In fact, in animals exposed to the higher concentrations of elastase used herein (15 and $20 \mathrm{IU})$, cholinergic responsiveness was negligible. The decreased magnitudes of cholinergic responsiveness were accompanied by decreased velocity of airway narrowing, which the present authors predicted would herald increased tethering forces within the tissue. It may be that elastase inhalation leads not only to the emphysematous changes in the parenchyma, but also to fibrotic remodelling of the airway walls per se. Enzymatically digested elastin is repaired by lung-derived cells [31], and even the first study using this rodent model [10] noted a net increase (by 30\%) in lung elastin levels in addition to the patchy emphysematous changes. BREWER et al. [32] have shown that alveolar walls exhibit more hysteresis following elastase treatment. Conversely, there may be collagen-related remodelling; ITO and co-workers $[33,34]$ found the airspace enlargement and loss of parenchymal elastin in this murine elastolytic model to be accompanied by increased (48\%) collagen content. It should also be acknowledged that the present experimental approach might bias against studying those slices/airways with the most emphysematous changes, due to airway collapse and tearing of the lungs during slicing, and instead predispose towards the study of airways which have become fibrotic (another feature of COPD that can be reproduced in this murine model), since they are more robust during the slicing procedure. It is also possible that the in vivo elastase injury includes a change within the smooth muscle itself, a loss of the contractile phenotype. Taking these factors into account, it is possible that the inflammatory and/or reparative processes that occur during the 2 weeks following elastase instillation induce a transition within the smooth muscle towards a more secretory phenotype (with concomitant loss of the contractile phenotype). The present authors discount the alternative explanation that elastase-treated tissues express more/different proteins on the alveolar walls, which make them more adherent to the glass surface used in the present study's in vitro apparatus, since the airway would be expected to be seen straining inwards (this has been the present authors' experience when airways were observed contracting against an agarose plug within the lumen) and/or portions of the airway seen tearing away suddenly from the glass. Neither was observed.

When the concentration-response relationship was examined for $\mathrm{ACh}$, no differences were found between the elastasetreated and naive tissues. As such, the inherent sensitivity of the ASM was not affected. One interpretation of these concurrent changes is that elastase exposure in vivo led first to tissue injury/destruction followed by a repair process, which left the airways scarred and fibrotic. The present authors are now adapting their histological assays in order to quantify elastin/collagen content in the very same thin slices from which videorecordings were made. Cutting of histological sections of these already very thin slices is difficult, and the agarose within the tissues may alter the staining assay per se. Alternatively, elastase treatment may have led to loss of ASM mass and/or otherwise compromised ASM function.

In contrast to the above, tissues pre-treated overnight with elastase exhibited the expected changes, i.e. tearing of the airway wall from the surrounding parenchyma, and slower and less complete relaxations back to resting diameter upon washout of ACh. Conversely, the magnitude and velocity of cholinergic contractions remained unaltered with respect to control slices. These observations are consistent with decreased tethering forces within the tissues brought on by the acute treatment with protease; elastin is vital for elastic recoil of the small airways and their ability to resist negative pressure collapse [35]. Overnight treatment with this protease would not have allowed sufficient time for tissue remodelling events, such as fibrosis or smooth muscle hyperplasia/hypertrophy (or transition of the latter towards the secretory phenotype). 
In conclusion, the present experimental approach directly allows visualisation of small airway narrowing with high spatial and temporal resolution while still in the native extracellular environment, providing a valuable insight into the kinetics of airway narrowing and the effects of altered tethering. Using the present approach, it was possible to discriminate differences in airway kinetics due to different degrees of smooth muscle activation $\left(10^{-7}\right.$ versus $\left.10^{-6} \mathrm{M} \mathrm{ACh}\right)$ or even strain of animal (Balb/C versus C57 mice; data not shown here). It is otherwise impossible to visualise airways of this size with the same resolution (e.g. computed tomography/ positron emission tomography). Nevertheless, the present technique does have some limitations. First, the absolute kinetics of airway narrowing could be affected by friction between the coverslip and the tissue slice, as well as by the agarose itself, which remains in the alveoli in a gel state throughout the experiment. However, these effects should be constant between treatment groups, and thus relative comparisons can still be made. Secondly, longitudinal forces are missing from the present experimental arrangement.

In future studies, care should be taken to examine the potential contributions of protease-activated receptors to the changes described herein. Also, it would be intriguing to extend this model to the human condition by comparing magnitudes and velocities of airway narrowing in lung slices obtained from surgical specimens of humans both with and without chronic obstructive pulmonary disease.

\section{ACKNOWLEDGEMENTS}

The authors would like to thank M. Sanderson and Y. Bai (Dept of Physiology, University of Massachusetts Medical School, Worcester, MA, USA) for their help with the lung slice technique.

\section{REFERENCES}

1 Baraldo S, Saetta M, Cosio MG. Pathophysiology of the small airways. Semin Respir Crit Care Med 2003; 24: 465-472.

2 Cosio MG, Majo J, Cosio MG. Inflammation of the airways and lung parenchyma in COPD: role of T cells. Chest 2002; 121: Suppl. 5, 160S-165S.

3 Janus ED, Phillips NT, Carrell RW. Smoking, lung function, and alpha 1-antitrypsin deficiency. Lancet 1985; 1: 152-154.

4 Pauwels RA. National and international guidelines for COPD: the need for evidence. Chest 2000; 117: Suppl. 2, 20S-22S.

5 Petty TL. COPD. Interventions for smoking cessation and improved ventilatory function. Geriatrics 2000; 55: 30-39.

6 Saetta M. Airway inflammation in chronic obstructive pulmonary disease. Am J Respir Crit Care Med 1999; 160: S17-S20.

7 Roy SK, Hu J, Meng Q, et al. MEKK1 plays a critical role in activating the transcription factor C/EBP-beta-dependent gene expression in response to IFN-gamma. Proc Natl Acad Sci USA 2002; 99: 7945-7950.

8 Fehrenbach H. Animal models of chronic obstructive pulmonary disease: some critical remarks. Pathobiology 2002; 70: 277-283.
9 Mahadeva R, Shapiro SD. Chronic obstructive pulmonary disease: Experimental animal models of pulmonary emphysema. Thorax 2002; 57: 908-914.

10 Valentine R, Rucker RB, Chrisp CE, Fisher GL. Morphological and biochemical features of elastaseinduced emphysema in strain A/J mice. Toxicol Appl Pharmacol 1983; 68: 451-461.

11 Stone PJ, Calore JD, Snider GL, Franzblau C. The dosedependent fate of enzymatically active and inactivated tritiated methylated pancreatic elastase administered intratracheally in the hamster. Am Rev Respir Dis 1979; 120: $577-587$.

12 Gray PR, Mitchell HW. Intramural elastase injection increases responsiveness of isolated bronchial segments. Pulm Pharmacol 1996; 9: 239-243.

13 Qian SY, Mitzner W. In vivo and in vitro lung reactivity in elastase-induced emphysema in hamsters. Am Rev Respir Dis 1989; 140: 1549-1555.

14 Celli BR. Pathophysiology of chronic obstructive pulmonary disease. Chest Surg Clin N Am 1995; 5: 623-634.

15 Siafakas NM, Vermeire P, Pride NB, et al. Optimal assessment and management of chronic obstructive pulmonary disease (COPD). The European Respiratory Society Task Force. Eur Respir J 1995; 8: 1398-1420.

16 Dandurand RJ, Wang CG, Phillips NC, Eidelman DH. Responsiveness of individual airways to methacholine in adult rat lung explants. J Appl Physiol 1993; 75: 364-372.

17 Dandurand RJ, Wang CG, Laberge S, Martin JG, Eidelman DH. In vitro allergic bronchoconstriction in the brown Norway rat. Am J Respir Crit Care Med 1994; 149: 1499-1505.

18 Bergner A, Sanderson MJ. Airway contractility and smooth muscle $\mathrm{Ca}^{2+}$ signaling in lung slices from different mouse strains. J Appl Physiol, 200395: 1325-1332.

19 Bergner A, Sanderson MJ. Acetylcholine-induced calcium signaling and contraction of airway smooth muscle cells in lung slices. J Gen Physiol 2002; 119: 187-198.

20 Bonniaud P, Kolb M, Galt T, et al. Smad3 null mice develop airspace enlargement and are resistant to TGF-beta-mediated pulmonary fibrosis. J Immunol 2004; 173: 2099-2108.

21 Bai Y, Zhang M, Sanderson MJ. Contractility and Ca2+ signaling of smooth muscle cells in different generations of mouse airways. Am J Respir Cell Mol Biol 2007; 36: 122-130.

22 Martin C, Uhlig S, Ullrich V. Videomicroscopy of methacholine-induced contraction of individual airways in precision-cut lung slices. Eur Respir J 1996; 9: 2479-2487.

23 Kott KS, Pinkerton KE, Bric JM, Plopper CG, Avadhanam KP, Joad JP. Methacholine responsiveness of proximal and distal airways of monkeys and rats using videomicrometry. J Appl Physiol 2002; 92: 989-996.

24 Adler A, Cowley EA, Bates JH, Eidelman DH. Airwayparenchymal interdependence after airway contraction in rat lung explants. J Appl Physiol 1998; 85: 231-237.

25 Duguet A, Wang CG, Gomes R, Ghezzo H, Eidelman DH, Tepper RS. Greater velocity and magnitude of airway narrowing in immature than in mature rabbit lung explants. Am J Respir Crit Care Med 2001; 164: 1728-1733.

26 Macklem PT. A theoretical analysis of the effect of airway smooth muscle load on airway narrowing. Am J Respir Crit Care Med 1996; 153: 83-89. 
27 Moreno RH, Hogg JC, Pare PD. Mechanics of airway narrowing. Am Rev Respir Dis 1986; 133: 1171-1180.

28 Lambert RK, Pare PD. Lung parenchymal shear modulus, airway wall remodeling, and bronchial hyperresponsiveness. J Appl Physiol 1997; 83: 140-147.

29 Shapiro SD, Goldstein NM, Houghton AM, Kobayashi DK, Kelley D, Belaaouaj A. Neutrophil elastase contributes to cigarette smoke-induced emphysema in mice. Am J Pathol 2003; 163: 2329-2335.

30 Barnes PJ. Mechanisms in COPD: differences from asthma. Chest 2000; 117: Suppl. 2, 10S-14S.

31 Stone PJ, Morris SM, Thomas KM, Schuhwerk K, Mitchelson A. Repair of elastase-digested elastic fibers in acellular matrices by replating with neonatal rat-lung lipid interstitial fibroblasts or other elastogenic cell types. Am J Respir Cell Mol Biol 1997; 17: 289-301.

32 Brewer KK, Sakai H, Alencar AM, et al. Lung and alveolar wall elastic and hysteretic behavior in rats: effects of in vivo elastase treatment. J Appl Physiol 2003; 95: 1926-1936.

33 Ito S, Ingenito EP, Arold SP, et al. Tissue heterogeneity in the mouse lung: effects of elastase treatment. J Appl Physiol 2004; 97: 204-212.

34 Ito S, Ingenito EP, Brewer KK, et al. Mechanics, nonlinearity, and failure strength of lung tissue in a mouse model of emphysema: possible role of collagen remodeling. J Appl Physiol 2005; 98: 503-511.

35 Starcher BC. Lung elastin and matrix. Chest 2000; 117: Suppl. 1, 229S-234S. 